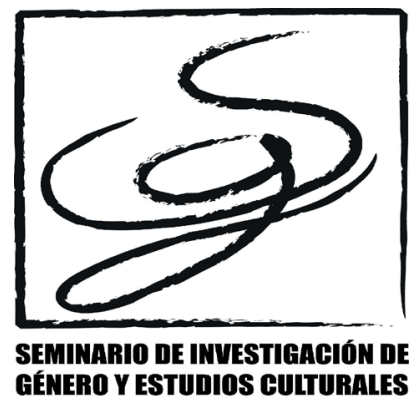

\title{
Agression sexuelle et réification de la femme dans quelques romans féministes camerounais
}

\author{
Sexual Assault and Reification of the Woman in some Cameroonian \\ feminist novels
}

Pierre Suzanne Eyenga Onana

Université de Yaoundé 1

eyonapiers@gmail.com

\author{
Fecha de recepción: 31/05/2020 Fecha de evaluación: 1/07/2020 \\ Fecha de aceptación: 24/11/2020
}

\begin{abstract}
:
The Cameroonian feminist novel is a combat novel. It acts as a form of advocacy that the writer formulates in order to postulate more dignified gender relations. In this novel, the demiurge fights for the advent of a more just social order at the heart of which all genders are equal and all sexes owe each other mutual respect. The novel works in several directions: to restore women's reputation in terms of rights and to allow them to dispose of their own bodies as respected human beings. However, this goal of reappropriating the female body runs up against a certain phallocratic vision of gender relations aimed at keeping women in the sexist bosom. Consequently, as a mode of declining sexual violence, does sexual aggression not participate in strategies whose aim is only the reification of women? By drawing inspiration from the sociocritical conceptual apparatus formalised by Pierre Barbéris and the gender approach, we answer this research question. The work is organized in three parts. In the first part, we examine the forms of violence against women in the feminist text under examination. The second part deals with the stylization of sexual violence. It questions the linguistic forms at work in the literary text, since the literary work is above all a work of art: beyond the reproduction of the observed facts, it is the production of language. The last part is concerned with the novelists' message or their vision of the world on the issue of sexual assault. The aim of this part is to show that rape is a serious violation of women's rights, a major obstacle to their empowerment and the affirmation of their identity.
\end{abstract}

Key-words: sexual assault; woman; feminist novel; sociocritic; phallocratic vision; gender approach; artwork; women's rights; empowerment; identity 


\section{Resumen:}

La novela feminista camerunesa es una novela de combate. Actúa como una forma de defensa que el escritor formula para postular relaciones de género más dignas. En esta novela, el demiurgo lucha por el advenimiento de un orden social más justo en cuyo centro todos los géneros son iguales y todos los sexos se deben respeto mutuo. La novela funciona en varias direcciones: restaurar la reputación de las mujeres en términos de derechos y permitirles disponer de sus propios cuerpos como seres humanos respetados. Sin embargo, este objetivo de reapropiarse del cuerpo femenino choca con una cierta visión falocrática de las relaciones entre los géneros que tiene por objeto mantener a la mujer en el seno sexista. Por consiguiente, como modo de disminuir la violencia sexual, ¿no participa la agresión sexual en estrategias cuyo objetivo es sólo la cosificación de la mujer? Inspirándonos en el aparato conceptual sociocrítico formalizado por Pierre Barbéris y en el enfoque de género, respondemos a esta pregunta de investigación. El trabajo está organizado en tres partes. En la primera parte, examinamos las formas de violencia contra la mujer en el texto feminista que se examina. La segunda parte trata de la estilización de la violencia sexual. Cuestiona las formas lingüísticas que actúan en el texto literario, ya que la obra literaria es ante todo una obra de arte: más allá de la reproducción de los hechos observados, es la producción de lenguaje. La última parte se refiere al mensaje de los novelistas o su visión del mundo sobre el tema de la agresión sexual. El objetivo de esta parte es mostrar que la violación es una grave violación de los derechos de la mujer, un gran obstáculo para su empoderamiento y la afirmación de su identidad.

Palabras clave: agresión sexual; mujer; novela feminista; sociocrítico; visión falocrática; enfoque de género; obra de arte; derechos de la mujer; empoderamiento; identidad

\section{Résumé}

Le roman féministe camerounais est un roman de combat. II agit comme une forme de plaidoyer que formule l'écrivain en vue de la postulation des rapports sociaux de sexe plus dignes. Dans ce roman, le démiurge lutte pour l'avènement d'un ordre social plus juste au cœur duquel tous les genres se valent et tous les sexes se doivent un respect mutuel. Ce roman travaille dans plusieurs sens: redorer le blason de la femme en matière de droits et lui permettre de disposer de son propre corps comme une personne humaine respectée. Cet objectif de réappropriation du corps féminin bute cependant contre une certaine vision phallocratique des rapports de genre visant à maintenir la femme dans le giron sexiste. Dès lors, comme mode de déclinaison de la violence sexuelle, l'agression sexuelle ne participe-t-elle pas des stratégies dont la finalité n'est que la réification de la femme ? En nous inspirant de l'appareillage conceptuel sociocritique formalisé par Pierre Barbéris ainsi que l'approche genre, 
nous répondons à cette question de recherche. Le travail est organisé en trois parties. Dans la première, nous scrutons les formes de violence dont la femme fait les frais dans le texte féministe examiné. La deuxième partie porte sur la stylisation des violences sexuelles. Elle questionne les formes linguistiques à l'œuvre dans le texte littéraire, puisque l'œuvre littéraire se veut avant tout une œuvre d'art: par-delà la reproduction des faits observés, elle est production de langage. La dernière partie se préoccupe du message des romanciers ou encore leur vision du monde sur la question de l'agression sexuelle. II s'agit dans cette partie de montrer que le viol constitue une grave enfreinte des droits de la femme, un obstacle majeur à son autonomisation et à l'affirmation de son identité.

Mots clés: agression sexuelle; femme; roman féministe; sociocritique; vision phallocratique; approche genre; œuvre d'art; droit de la femme; autonomisation; identité

\section{Introducción}

"L'un des problèmes majeurs qui a toujours prévalu dans les sociétés humaines est la question des rapports entre les deux sexes hommes et femmes-» (N'guessan, 2017: 3). Ce postulat interpellateur souligne la complexité de la vie en société et montre que le jeu social n'est pas aisé pour tous les acteurs appelés à se frotter les uns aux autres au gré de leurs diverses humeurs conditionnées par certaines pesanteurs. Dans la société patriarcale, par exemple, la femme est présentée comme un éternel valet au service de l'homme: elle est née pour le servir en tout temps et en tout lieu, sans jamais rechigner. Au regard d'un tel contexte, fondé sur l'essentialisme ${ }^{1}$, Simone de Beauvoir dira que «la femme ne pense pas sans l'homme, elle n'est rien d'autre que ce que l'homme décide qu'elle soit» (1949: 16). Cette vision réductrice des rapports de sexe, qui bien entendu ne reçoit pas l'aval de la théoricienne De Beauvoir, lui fait dire, en ironisant, que "le mâle apparaît comme infiniment privilégié: sa vie génitale ne contrarie pas son existence personnelle» (1949: 79). Ces points de vue, qui confortent le statut de mâle dominant que s'octroie l'homme chaque fois qu'il a affaire à la femme dans un espace de vie régi par la phallocratie, ont suscité la prise de parole des femmes aux fins de tenter d'infléchir la logique sous-tendant les rapports de sexe et de genre. Le féminisme déploie ainsi son dard, sous la forme d'un ensemble d'idées politiques et philosophico-sociales, afin de chercher à promouvoir les droits des femmes et leurs intérêts dans la société civile.

L'une des violations desdits droits consiste à contraindre une femme à entretenir des rapports sexuels non consentis avec un inconnu, tout juste parce que ce dernier se trouve être un homme. L'agression sexuelle participe ainsi des stratégies mises sur pied par les phallocrates

\footnotetext{
1 Cette approche des rapports sociaux de sexe consacre le primat de l'essence sur l'existence. Elle ne suppose pas de libre arbitre de l'individu alors considéré comme le produit de déterminismes qui le définissent et dont il ne peut s'en extraire.
} 
aux fins de réifier la femme. Voilà pourquoi le roman féministe s'inscrit dans la dynamique de visualiser «la conscience d'appartenir à une classe majoritaire qui ploie sous le joug des pratiques barbares; (...) d'être chosifiée ${ }^{2}$ et refuser d'être considérée comme un objet sexuel ou une machine à procréer "(Beyala,1995: 11). Dès lors, comment s'opère la dénonciation des agressions sexuelles subies par la femme en vue de faire prendre conscience aux hommes des rapports parfois houleux qui les lient?

La sociocritique de Pierre Barbéris et l'approche genre soustendent notre réflexion. Telle que définie par Edmond Cros, la sociocritique «ne s'intéresse pas à ce que le texte signifie mais à ce qu'il transcrit, c'est-à-dire à ses modalités d'incorporation de l'histoire, non pas d'ailleurs au niveau des contenus mais au niveau des formes " (2003: 53). Barbéris la conçoit comme une démarche holistique en ceci qu'elle couvre plusieurs domaines de recherche. La sociocritique désigne alors
La lecture de l'historique, du social, de l'idéologique, du culturel dans cette configuration étrange qu'est le texte: il n'existerait pas sans le réel, et le réel à la limite, aurait existé sans lui; mais le réel, alors, tel que nous pouvons le percevoir, serait-il le même, exactement? (Barbéris, 1990: 153).

Le mode opératoire de Barbéris que nous adoptons dans le cadre de cette étude s'articule autour de deux axes: la lecture de l'explicite et la lecture de l'implicite.

S'agissant de l'approche genre, la variante que nous appliquons est l'antinaturalisme féministe. Cette approche critique vise à «en finir avec la nature en réduisant totalement le biologique au socioculturel (car) la biologie du sexe est bien plus plastique que la politique du genre » (Krauss, 2005: 39). Pour Krauss, «il y a naturellement des différences entre les sexes mais ces différences ne sont pas significatives» (2005: 39). Le postulat kraussien vise à dire que les différences sus-évoquées entre les sexes ne devraient pas justifier un quelconque écart de conduite de la part des hommes.

Le travail s'organise en deux étapes. La première décrypte les formes d'agression sexuelle que subit la femme dans le texte féministe. On montre à chaque fois que c'est le regard essentialiste qui pèse sur la femme qui en conditionne l'ostracisation par l'homme. La dernière partie se subdivise en deux sous parties. La première traduit l'idée que «par le travail de l'écriture, (le texte de roman) produit un autre sens» (Mitterand, 1980: 7). II s'agit de scruter la fonction productive à l'œuvre dans le roman en illustrant comment l'écrivain met en exergue sa compétence narrative car, «la littérature n'est bien qu'un langage, c'est-à-dire un système de signes: son être n'est pas dans son message, mais dans ce système» (Barthes, 1964: 257). La dernière sous partie s'intéresse davantage au message des féministes sur la question de l'agression sexuelle, étant

${ }^{2}$ C'est l'auteure qui souligne 
donné que tout roman se veut en réalité un «discours sur le monde» (Mitterand, 1980: 5).

\section{La lecture de l'explicite: typologie des agressions sexuelles}

La sociocritique est un mouvement qui «s'opère (...) à partir d'une recherche et d'un effort tâtonnant et découvreur qui invente un nouveau langage, fait apparaître de nouveaux problèmes et pose de nouvelles questions» (Barbéris, 1990: 154). L'un de ses axes de déploiement est l'explicite. A travers ce concept, le théoricien entend «recharger le texte de ce qui y est déjà, mais qui a été marginalisé ou évacué. II ne s'agit pas ici d'une symbolique obscure mais de références claires à restituer, et qui peuvent être disséminés» (Barbéris, 1990: 168). En d'autres termes, dans la quête de l'explicite, il importe de " traquer ce qui, dans le texte, se trouve dit $^{3}$ et dénoté, et de travailler dans deux directions: relecture du texte et critique des non-lectures et de leurs raisons» (Barbéris, 1990: 169). Dans les textes féministes en examen, l'agression sexuelle épouse plusieurs variables: l'inceste, le viol et la pédophilie.

\subsection{L'inceste, une néantisation des valeurs éthiques familiales}

L'inceste s'avère une forme de transgression des valeurs traditionnelles qui fondent la culture africaine millénaire. Suivant un ensemble de normes qui régissent la famille africaine, il a ainsi été institué un mode de conduite qui interdit aux frères et sœurs d'entretenir des rapports sexuels au nom des liens filiaux sacrés qui les unissent pour la vie. C'est pour cela que Pehmba, dans le roman éponyme de Louis-Marie Ongoum, décède le jour même de la nuit de ses noces avec Pondah, son frère, né de l'union adultérine entre Nlombob, son père, et la mère de son ami. Elle n'aura pas consommé son mariage. La répulsion nourrie à l'endroit de Pondah, garçon brave et admirable, procède de ce que Pehmba ignore avoir affaire à son frère que lui recommande pourtant fortement sa mère. Elle souffre dans son corps pour s'être inconsciemment amouraché de son frère. Et cela se voit tout au long de leur semblant d'idylle. La forme de violence subie par Pehmba résulte d'une forme d'inceste psychologique parce que la fille est presque offerte à son frère pour jouer le rôle de son épouse. Autant affirmer que les relations sexuelles connaissent des limites dès lors qu'il s'agit de frères.

De même, dans Le fruit défendu, on est en présence d'une forme de sexualité qu'on pourrait qualifier d'excentrique. Elle brise les normes socioculturelles et religieuses établies au bénéfice de l'homme dont elle sert la cause. La sexualité ne se pratique pas entre cousins comme tel est le cas dans le roman évoqué. Amoureux de sa cousine Mengue Rose, Guillaume Alima s'entend opposer une fin de non-recevoir. Sa cousine lui répond que l'idylle manifestée à son endroit ne peut prospérer d'autant que l'amour qu'elle lui voue est celui que nourrit une sœur pour son frère. Elle le ramène sur terre à travers ces mots qui font réaliser la malédiction qui pèserait sur eux s'ils venaient à poser un acte coupable: «d'ailleurs tu

\footnotetext{
${ }^{3}$ C'est l'auteur qui souligne chaque fois que dans la citation il y a usage de l'italique.
} 
ne trouveras nulle part dans ces tribus une fille assez folle pour t'écouter. C'est strictement défendu. Tu n'y trouveras que malheur et colère des dieux et des hommes» (Ahanda Essomba, 1975: 50).

Dans la société traditionnelle africaine, la forme d'éducation privilégiée est de type socio-culturel. L'enfant qui naît et grandit subit une formation au sein de la communauté. L'éducation de base qu'il reçoit en fait un homme respectueux des valeurs prônées par sa culture. L'accent est ainsi mis sur le respect scrupuleux de la parenté afin d'éviter d'éventuels désagréments. Le Fruit défendu métaphorise la sexualité interdite entre des frères comme le relève Mengue Rose: "Guillaume tu perds la tête. Tu sais que c'est défendu de parler ainsi... Tu ne peux pas m'aimer dans le sens où tu l'entends. J'en mourais de honte. C'est défendu. Comprends-tu? II faut m'aimer comme une sœur» (Ahanda Essomba, 1975: 53). L'usage dans cet exemple de l'interrogation rhétorique doublée du discours injonctif introduit par «il faut», attestent de la portée éthique d'une pratique sexiste visant à définir la femme comme «un être né à genoux aux pieds de l'homme» (Beyala, 1995, 11). Fondant ses désirs sur ce postulat, Guillaume Alima drogue et viole sa cousine Mengue Rose. Le rapport de force entre les deux sexes étant à l'avantage de l'homme, la femme cède à la brutalité phallocratique et se laisse violer contre son gré. Le récit du narrateur relate ainsi la violence des faits: " la lutte dura longtemps, Alima déchirait les vêtements de Mengue avec une rage folle. Dans un ultime effort, il la renversa» (Ahanda Essomba, 1975: 72). La folie du mâle dominant n'est pas moins décrite à travers ces propos qui traduisent la violence machiste mais surtout l'incapacité physique de la femme à riposter contre les attaques lancées contre sa personne: «épuisée, Mengue ferma les yeux, à moitié évanouie pendant que son cousin s'acharnait sur elle avec toute la fougue des instincts déchaînés» (Ahanda Essomba, 1975: 72). Lorsque la forme de la violence met en opposition une petite fille et un homme très âgé, on parle de pédophilie.

\subsection{La pédophilie, un acte de violence contre la petite fille}

Le code pénal en vigueur en France depuis 1994 définit l'agression sexuelle comme toute atteinte sexuelle commise avec violence, contrainte, menace ou surprise. La pédophilie s'inscrit dans cette forme de violence. II s'agit "d'un épanchement érotique pour les enfants» (Chesneau, 2010: 59). Krafft-Ebing ajoute que cette pratique sexuelle traduit chez l'homme âgé une préférence sexuelle pour les enfants, généralement d'âge pubère ou en début de puberté. Généralement, avant d'entretenir des relations sexuelles, deux partenaires conviennent de passer à l'acte suivant certains préalables. Mais il est important, pour ce faire, que la jeune fille ait atteint l'âge de la majorité civile avant de pouvoir goûter au plaisir de la vie sentimentale. Tel n'est pourtant pas le cas dans les romans étudiés, au regard de l'âge des partenaires. Dans La Poupée Ashanti de Francis Bebey, tout comme Sous la cendre le feu d'Evelyne Mpoudi Ngolle, l'âge de la partenaire et son consentement naiff obligent à évoquer un acte de pédophilie. La jeune fille ignore ce qu'elle vit ou 
encore ce qu'on lui fait endurer. Et c'est lorsqu'elle le raconte à sa mère, de façon inconsciente, qu'elle permet au lecteur de mesurer l'ampleur de la violence sexuelle subie. Dans La Poupée ashanti, Edna sera la victime résignée de son maître d'école Bunefo. Chargé de lui faire des cours de répétition après les classes, ce dernier en profite pour mettre à exécution son plan pédophile. II prend l'habitude d'amener chez lui la petite fille et de la soudoyer avec des bonbons afin de mieux voiler sa ruse. La victime raconte à Mam, sa grand-mère qui lui tient lieu de mère: «j'y suis allée (...) plusieurs fois, surtout les après-midi où tu n'étais pas là (...) Oh, il était gentil, et la première fois, il m'a donné des bonbons» (Bebey, 1973: 50). Croyant avoir affaire à un homme responsable, Edna perd sa virginité comme le révèle cet aveu empreint de pudeur et de naïveté à sa grandmère: «il m'a dit ... que cela... fait mal au début. Mais après ça va mieux» (Bebey, 1973: 50). II va sans dire que dans cette expérience sexuelle, Edna en ressort perdante bien qu'ayant marqué son accord de principe pour la tenue de l'acte sexuel. Car, s'il est vrai qu'on peut acheter des bonbons, il apparaît que la virginité se perd une fois pour toutes. La violence sexuelle induit une autre forme de perte: celle de son instruction. Car, après ce viol, la grand-mère d'Edna rompt le contrat de travail qui lie sa petite-fille à Bunefo en la consignant à ses côtés comme vendeuse du marché.

Dans le même ordre d'idées, la recherche par l'homme du mieuxêtre l'entraine parfois à poser des actes condamnables ou qui transgressent les normes du code éthique. Désireux d'améliorer ses conditions de vie, ce dernier n'hésite pas à sacrifier la virginité de sa progéniture à l'autel de la cupidité et de l'égoïsme. Dans Sous la cendre le feu, Djibril cède aux conseils déstabilisateurs de ses amis et préfère suivre la conduite édictée par un féticheur afin de devenir riche. La condition pour le devenir effectivement consiste à violer sa propre fille. On est en face d'un cas mitigé qui vogue entre inceste et pédophilie. C'est par hasard que la mère de Mina sera mise au courant de l'amour coupable entre fille et père dans un cadre diégétique que Genette (1972) identifie comme la scène ${ }^{4}$. Croyant avoir affaire à un être aimable, la jeune fille étale sa naïveté sans toutefois se rendre compte de la gravité des faits relatés:
Maman ce dont vous parliez tout à l'heure c'est quoi?
Comment ce dont nous parlions?
Quand vous parliez, tes amis et toi des choses qu'un homme peut faire avec une fille... (...)
Ça veut dire quoi violée?
Heu.... C'est quand un homme couche avec une femme en la forçant (Mpoudi Ngolle, 1990: 194-195).

La réaction de Fanny, la petite fille en question, atteste de son ignorance et de sa naïveté lorsque sa mère lui reprécise qu'il y a des préalables avant qu'un homme ne couche avec une femme. Le critère

\footnotetext{
${ }^{4}$ II s'agit d'un dialogue sans intermédiaire au cours duquel temps du récit et temps de la narration sont isochrones
} 
d'âge est justement le premier comme l'asserte Mina dans une veine marquée par le discours injonctif: "je tiens à te dire que si jamais un homme te parle de ces choses-là, tu dois l'éviter. Quand tu seras grande, (...) Mais avant tu ne dois laisser aucun garçon, aucun homme te toucher» (Mpoudi Ngolle, 1990: 195-196). Mais l'auto-culpabilisation de Fanny relève du tabou et commence lorsque celle-ci hésite à s'exprimer en présence de sa mère. Elle confiera finalement son erreur dans une scène forte:

Même si cet homme est mon père?

Fanny pourquoi me demandes-tu ça?

Parce que ... parce que papa l'a fait avec moi, un vendredi, quand j'étais avec lui à l'étude (Mpoudi Ngolle, 1990: 196).

A son tour, Mina avoue au docteur Lobe, en des termes plus clairs, qu'elle vient de trouver la clé de sa guérison: «mon mari a violé ma fille (...) oui il a violé ma fille, Fanny» (Mpoudi Ngolle, 1990: 193).

N'ayant même pas réalisé la gravité de l'acte posé, elle en parle à sa mère dans un naturel déconcertant. Le narrateur le relève en affirmant: «Fanny haussa les épaules, montrant par là qu'elle ne voyait pas grand mal au fait qu'un homme se couche dans le même lit qu'une femme» (Mpoudi Ngolle, 1990: 194-195). La violence subie par la femme peut, en outre, revêtir la forme du viol perpétré à son endroit par des personnes sans foi ni loi.

\subsection{Le viol, une violation du droit de la femme à l'intimité}

D'une manière ou d'une autre, violer, c'est voler. Aussi, violer une femme revient-il à lui voler son intimité, sa dignité et parfois ses biens matériels. Les agresseurs, qui sont en réalité des voleurs ambulants, mettent en œuvre diverses stratégies visant à déposséder leurs victimes de leur avoir. Contrairement à Djibril qui use de stratagèmes malsains pour progresser financièrement, Francine, qui vient d'obtenir un poste dans la société TNT, subit un choc violent du fait qu'elle se fait violer un soir alors qu'elle essaie de rallier son domicile en passant par une voie dérobée. Le narrateur omniscient relate qu'«après l'avoir jeté au sol, son agresseur entreprend de fouiller son sac à main jusqu'à la déposséder de tout son contenu» (Ngo Iboum, 2011: 38). La particularité du viol de Francine réside dans le fait qu'elle perd à la fois ses téléphones et son argent en plus de sa dignité de femme libre. Le traumatisme psychologique qu'elle subit procède de ce qu'elle se trouve en face de huit bandits comme le lui rappelle le chef des agresseurs: "tu as intérêt à coopérer» (Ngo Iboum, 2011: 38). La violence physique se double ainsi d'une variante verbale dont la caractéristique est le dialogue de sourd qui s'instaure entre la victime et ses bourreaux. Bien que la victime feigne d'être enceinte, ses supplications n'émeuvent pas outre mesure ses agresseurs. La violence de la réaction des bourreaux se voit à travers ces mots: «tu as un petit ami et tu es enceinte. Ça tombe bien car cela signifie que tu es déjà expérimentée en matière de sexe alors je veux que tu te 
déshabilles et que nous fassions l'amour. Dépêche-toi» (Ngo lboum, 2011: 39).

Faire l'amour se révèle un geste qui traduit la communion de cœurs entre deux êtres qui s'aiment. Mais parler d'amour, dans le cadre d'un viol, en rajoute à la peine psychologique et à l'horreur de la victime. Fanny s'indigne de ces mots: "faire l'amour! Quelle horreur! C'est ainsi qu'il voyait la chose. Nous devions faire l'amour. Pour lui c'était tout sauf un viol» (Ngo Iboum, 2011: 39). En déclarant par la suite, «il me viola» (Ngo Iboum, 2011: 40), la jeune femme traduit toute la charge de la violence qui marque son esprit. On le voit, l'arme du crime pour le bourreau aura été sa force physique. Elle contribue à faire plier sa victime à sa volonté.

Par-delà la figuration des réalités observées, c'est-à-dire leur reproduction à travers le miroir stendhalien, force est de relever leur production au moyen de la langue par la compétence narrative et linguistique de l'écrivain. II s'agit de montrer que «la forme (au-delà comme en deçà de la phrase, s'entend) est une médiation naturelle entre la substance sociale, extratextuelle, et le sens que prend l'énoncé romanesque» (Mitterand, 1980: 17).

\section{L'implicite ou la mise en forme du récit de la violence}

Par implicite, Barbéris entend démontrer qu'un «texte n'est pas fait de choses en clair et qu'on n'avait pas pu ou pas voulu voir. Un texte est aussi une arcane qui dit le sociohistorique par ce qui peut ne paraître qu'esthétique, spirituel ou moral» (1990: 169). Dans les récits en examen, l'implicite se dévoile à travers les axes qui suivent: les transgressions formelles; les niveaux de langue, l'hybridité langagière et la rhétorique de la violence.

\subsection{Les transgressions formelles}

Elles visent à montrer comment un «texte transgresse un art poétique» (Barbéris, 1990: 141). Autrement dit, à travers l'examen des transgressions formelles, on montre que «les querelles littéraires portent toujours sur le style que ce soit la construction d'une phrase, les niveaux à la prosodie, la construction d'une intrigue, la construction des personnages ou les niveaux de langue» (Barbéris, 1990: 141). Dans les textes étudiés, le narrateur adopte deux modes de présentation de la problématique de l'agression sexuelle: les anachronies narratives, l'intergénéricité et l'usage de figures de rhétorique.

\subsubsection{Les anachronies narratives}

Dans la typologie de Genette, les anachronies narratives soulèvent la question de l'ordre du récit. Le démiurge peut en effet ponctuer celui-ci de retours en arrières ou de projections en avant dépendamment de sa sensibilité. Les anachronies se présentent sous deux formes: les analepses et les prolepses. L'analepse est «l'évocation après coup d'un événement antérieur au point de l'histoire où l'on se trouve» (Genette, 1972: 82); tandis que la prolepse décrit «toute manœuvre narrative 
consistant à raconter ou évoquer d'avance un événement ultérieur» (Genette, 1972: 82).

Dans La Poupée Ashanti par exemple, on note qu'une rétrospection a cours dans le récit du viol d'Edna par son directeur d'école lorsque la petite fille expose à sa grand-mère et à sa tante les difficultés auxquelles elle fait face pour apprendre à lire et à écrire dans sa classe. En effet, Edna déplore les méthodes pédagogiques inappropriées de son maître et entend susciter une confrontation entre sa grand-mère et l'éducateur Bunefo. Face à la réticence de sa parente, Edna justifie son geste inconscient en ressassant se rapports avec l'enseignant mis en cause. Elle étale au passage les atouts qu'offrait à son sens son éducateur dans une veine rétrospective: «il m'a dit: tu es jolie, Edna, et je t'aime beaucoup. Viens avec moi, je vais te montrer chez moi» (Bebey, 1973: 49). Le récit premier portant sur la pédagogie est ainsi interrompu pour un instant pour céder la place à un autre qui s'opère sous la forme d'une digression.

Dans Sous la cendre le feu, l'ensemble du récit fonctionne sous le mode d'une vaste analepse, exactement comme l'est le récit du viol de Francine dans Fleur brisée. Les deux récits sont achevés au moment où le narrateur entreprend de les relater. Dans le premier cas, Mme Mohammadou raconte au Docteur Edimo l'ensemble des péripéties qu'elle a essuyées dans le cadre d'une thérapie secrète mise sur pied par le psychiatre. II s'agit pour le médecin d'activer les souvenirs de sa patiente afin d'y déceler l'indice fatal ayant conduit à sa dépression. De nombreux événements se bousculent dans son esprit. C'est par hasard qu'elle est interrompue par sa fille Fanny qui lui pose de biens curieuses questions dans un arrière-fond de naïveté: «maman, ce dont vous parliez tout à l'heure, c'est quoi? (...) Quand vous parliez, tes amies et toi, des choses qu'un homme peut faire avec une fille» (Mpoudi Ngolle, 1990: 194). Le récit des épisodes de la vie de Mina est momentanément suspendu pour laisser libre cours à des digressions sur le viol. Et c'est dans cette vague narrative que Mina est mise au courant du viol de sa fille par son père.

S'agissant de la prolepse, elle s'offre à lire dans Fleur brisée et $L e$ Fruit défendu. Dans le premier roman, le narrateur commence par mettre le lecteur en face d'une scène de viol sans au préalable le situer sur les circonstances ayant conduit à cet incident dans la vie de l'héroïne de Ngo Iboum. La prospection consiste pour le narrateur à introduire un événement du récit qui sera par la suite repris dans le roman. Pour davantage montrer qu'il s'agit d'une anticipation sur le cours logique de la narration, la romancière intitule son chapitre «ma vie d'avant» (Ngo lboum, 2011: 12). Dans ce chapitre, la narratrice relate sa vie telle qu'elle se présentait avant le jour fatal de son viol. Ce n'est qu'au chapitre deux, «le jour où tout a basculé», que le lecteur réalise qu'il bénéficie d'un avantgoût du récit, avant même qu'il n'ait lu l'ensemble des 115 pages du roman.

Dans le second roman, le plan d'Alima de violer sa cousine ne survient pas de façon subite. II l'a planifié avant de passer à l'acte. 
Sentant que sa cousine lui file entre les doigts, parce que désormais promise à Jacques, il la taxe de fille-facile parce qu'il juge qu'elle a rapidement consenti à se marier. II planifie alors son ainsi que le montre ce fragment: "la deuxième solution c'était le viol, mais au bout, il y avait le scandale (...) oui, le viol» (Ahanda-Essomba, 1975: 68). Une fois conçue dans sa conscience, l'idée prend peu à peu corps puisqu'Alima finit par la réaliser dans la suite du récit; notamment à la fin du chapitre 6. La narration des violences subies par les femmes s'opère également au moyen de l'intergénéricité ou hybridité langagière.

\subsubsection{L'intergénéricité, une variante de l'hybridité du style}

Jacques Chevrier dit de l'hybridité générique qu'elle est la règle pour les œuvres postcoloniales«dans la mesure où les catégories habituelles (roman, poésie, théâtre ...), héritées des modèles occidentaux, font de plus en plus l'objet d'une remise en question» (1989: 232). S'appuyant sur les travaux de Robert Dion, Frances Fortier et Elisabeth Haghebaert, François Harvey identifie trois processus d'interaction générique: La différenciation, l'hybridation et la transposition. Nous ne nous intéressons qu'au phénomène de l'hybridation. II se définit comme «la combinaison de traits génériques hétérogènes dans une même œuvre» (Harvey, 2012: 128). De nombreux genres foisonnent dans notre corpus. Nous mettons l'emphase sur les genres oral, épistolaire, le témoignage et le journal ou chronique.

Le genre oral se distingue à travers l'usage de parémies qui agrémentent la réflexion entre personnages dans un contexte argumentatif. Elles ressortent l'identité du texte littéraire examiné. Dans $L e$ Fruit défendu, alors qu'il rejette la faute commise par ses soins, Guillaume Alima est ramené à la raison par son père Mbida qui utilise ce proverbe: «l'éléphant qui marche dans la forêt laisse les tracesde ses pas, l'arbre fruitier produit des fruits, l'homme est appelé à procréer pour continuer la création et pour perpétuer la lignée " (Ahanda-Essomba, 1975: 142). Devant l'obstination d'un Alima opposé à l'idée de prendre part au rite visant à expier le vice commis, son père renforce son argumentaire par ces mots: "on ne change pas en un jour toute une civilisation» (AhandaEssomba, 1975: 142). Ces ingrédients issus de la tradition africaine sont de nature à renforcer le processus d'argumentation chez un père soucieux de convaincre son fils afin que ce dernier se soumettre à la loi ancestrale. Le père ira même jusqu'à introduire un conte dans son propos aux fins de ramener à la raison son fils têtu.

Le genre épistolaire laisse penser à la lettre. Ce moyen de communication permet à Kono, le frère de Mbida, d'exposer sa fille à l'éducation de son oncle. Dans la lettre, le message se veut clair: «tiens! Voici la lettre de ton "frère" Kono. C'est toi qui es responsable de la petite, moi je ne suis jamais là» (Ahanda-Essomba, 1975: 146). C'est donc à travers la lettre de son frère Kono que Mbida introduit «sa» fille Mengue à son frère Alima. Cette lettre se révèle un agent catalyseur essentiel parce qu'il déclenche le viol de Mengue. C'est effectivement au premier instant de sa rencontre avec sa sœur qu'Alima s'en est fortement épris. La lettre 
énonce pourtant l'objectif fixé par toutes les parties. Son contenu n'a, à l'origine, aucune visée malsaine. Le viol qu'engendre par la suite ce moyen de communication résulte du désir profond de Mbida de présenter la jeune fille à celui qu'elle croyait être son frère:

Cher frère

Je t'envoie ta fille Rose comme convenu. Elle a été élevée à la mission catholique. Elle est obéissante et laborieuse. (...) Veille sur elle comme tu as fait pour tes filles dont j'entends les éloges jusqu'ici

Ton frère Kono (Ahanda-Essomba, 1975: 47).

Le témoignage se révèle un genre intermédiaire puisqu'il se situe entre le genre écrit et le genre oral. II consiste pour un personnage à se donner en exemple dans une situation de vie de violée afin de permettre à une victime de tenir le coup. Tel est le cas de l'héroïne de Fleur brisée qui reçoit un témoignage de la part de la voisine à Niès, sa grande sœur, peu de temps après son viol. Son témoignage vise à lui remonter le moral. L'expérience partagée marque la narratrice lorsque celle-ci reconnaît: "elle avait compris ce qui se passait à cause de l'état de mes vêtements, mon slip dépassait mon jean qui était à l'envers et dont le bandit avait fait sauter les boutons (Ngo Iboum, 2011: 43). Ces détails montrent que l'interlocutrice jouit d'une expérience de femme violée, parce qu'elle ne panique pas comme le reste du voisinage. La narratrice confie qu'«elle n'avait pas peur de moi ce soir-là» (Ngo lboum, 2011: 43). II convient d'affirmer que le témoignage de la voisine agit efficacement sur la victime de l'heure. II produit l'effet de premiers soins psychologiques prodigués à un malade amené aux urgences. II permet de consoler la victime surtout que la voisine l'accompagne d'autres gestes tels que l'appel lancé au copain de la victime. Se sentant soutenue, la victime amorce la rédaction de son journal.

La chronique ou journal assure une fonction thérapeutique dans Fleur brisée. II s'agit pour la narratrice de panser les blessures du viol restées béantes dans sa mémoire. Cette dernière entreprend de guérir personnellement de son drame en couchant sur du papier les moments terrifiants qui articulent la profondeur de son malaise une fois le viol subi. L'idée du journal s'inscrit dans cette dynamique. II s'agit d'une chronique permettant à la narratrice de passer en revue les moments phares de sa vie à travers un miroir quotidien. Elle amorce sa narration le 14/09 en précisant: «cela fait vingt-trois jours exactement que j'ai été violée (23/08 14/09). Je reviens de chez le docteur que j'ai connu grâce à une adorable grande sœur» (Ngo Iboum, 2011: 65). Le journal s'étend du chapitre 4 au chapitre 5 , au moment où le récit s'érige en une instance de conseils. La narratrice apparaît comme requinquée et bercée dans un élan de dépassement courageux. Elle énonce alors quinze conseils destinés aux victimes de viol. L'épilogue du récit se révèle également une chronique. C'est à cet instant que le lecteur apprend la coïncidence entre l'âge de la narratrice et celui de la romancière. Ecrit à la première personne du singulier, le roman s'offre dès lors comme la biographie d'une femme violée. La chronique s'achève un 24/03 lorsque la narratrice dévoile au 
lecteur son âge: «j'ai vingt-quatre ans aujourd'hui» (Ngo Iboum, 2011: 115).

Au regard de l'examen des questions de forme qui précède, force est de convenir que «le roman n'a pas à charge de représenter fidèlement le réel, mais de le recréer et de l'investir de signification» (Onomo, 1998: 81). L'analyse de la signification du récit constitue la matière de la dernière partie de notre étude. Elle revient à disséquer la vision du monde des romanciers étudiés.

\section{Le roman féministe: un discours sur le monde}

Précisant le rôle du romancier dans la création de la littérature, Onomo ajoute: «il choisit, élude, intensifie et la sélection du narrateur dans le monde réel est commandée par l'idéologie du groupe auquel il appartient» (1998: 81-82). Les auteurs féministes constituent les membres du groupe en question. Si pour eux, il s'agit dans un premier temps d'établir que certaines pesanteurs sexistes ont la peau dure, force est de souligner que leur attitude n'a rien de défaitiste. II s'agit surtout de démontrer que «la domination masculine est encore trop présente dans le monde, même si elle s'est déguisée. La machine phallocratique est graissée, parfaite» (Beyala, 1995: 77). II revient donc aux femmes «de la démolir. Sans peur, sans culpabilité mais sans agressivité, en réclamant leurs droits sans honte de déplaire» (Beyala, 1995: 77). Les femmes violentées entendent donc dans un premier temps se réapproprier leur corps avant de formuler leur plaidoyer afin que poigne à l'horizon une vision constructionniste des rapports de sexe.

\subsection{Militance pour une réappropriation du corps féminin}

La réappropriation du corps par la femme consiste pour cette dernière à reprendre en mains son destin en impulsant des stratégies diverses visant à la libérer de l'hydre du mal dominant. On pense, dans cette logique, à Ken Bugul et son roman Le Baobab fou, où elle s'attaque à la question de la virginité de la femme alors que cet attribut se perd très souvent par les soins de l'homme. Perdre sa virginité, hors mariage, serait pour la femme un motif de scandale et un déshonneur pour toute sa famille. Voilà pourquoi la romancière subvertit cette façon de penser et s'insurge contre le contrôle de virginité qu'on fait subir à la femme. Ce test s'avère humiliant et pour le moins ridicule tant il ne constitue en rien un critère de vertu fiable chez la femme lorsqu'on sait que dans son cas, celle-ci perd sa virginité à la suite d'une agression sexuelle organisée par son instituteur: «je repensais à tout ce qui m'était arrivé depuis que ma virginité qui me rattachait à toute une génération s'était envolée avec mon professeur d'histoire» (Ken Bugul, 2009: 73). Dans Femme infidèle, Talahatou milite en faveur de la pénalisation de l'excision. Ramant à contre-courant des femmes d'un autre âge qui soutiennent que «l'excision fait l'honneur de la femme. Elle permet d'éviter les injures de tes coépouses, car le clitoris est considéré comme une impureté» (Sadamba, 1988: 22), elle se réapproprie son corps vilipendé sans son aval en optant pour la prostitution. Talahatou traduit sa haine contre un système 
relationnel qu'elle juge obsolète parce que réifiant la femme et lui imposant, de surcroit, un époux polygame ayant l'âge de son père. Talahatou institue son infidélité comme une forme d'humiliation de l'homme et s'habille librement au mépris des préceptes du code vestimentaire musulman. C'est une femme mentalement libérée qui affirme: «je passai la nuit chez le policier sans me soucier le moins du monde de Morou qui devait sillonner les rues de Lomé à ma recherche» (Sadamba, 1988: 50).

De même, dans Le Journal intime d'une épouse, le principe de la sororité amène certaines épouses violées du Traquenard à se mobiliser dans le cadre d'un front commun bâti autour de la pratique du lesbianisme. Le refuge dans cette pratique conforte à l'idée que la violence est d'essence masculine et, en tant que telle, peut être conjurée pour peu que la femme y oppose une option d'amour en vue de la contrecarrer. Félicité et Merveille pensent ainsi trouver une alternative à la violence sexuelle qui leur vole leur dignité et les rend mère par les soins des chauffeurs-violeurs de leur époux absentéiste. La première soutient: «les femmes, ça peut dépanner quand les hommes sont vilains. (...) Je suis bisexuelle et, en attendant, activement lesbienne. (...). En baisant l'une avec l'autre, on ne risque pas de tomber enceintes" (Bonono, 2007: 38).

Toutefois, la réappropriation du corps de la femme va plus loin dans le texte de Bonono car la femme utilise la violence afin d'opposer une fin de non-recevoir à cette pratique. Victime de viol par Balthazar-Gaston, l'un des chauffeurs-violeurs à la solde de l'époux légitime, Sophie finit par prendre sa revanche sur ce dernier en lui ôtant la vie pendant son sommeil. La narratrice omnisciente relate ainsi les faits: «BalthazarGaston a été trouvé mort chez Sophie, la nouvelle épouse de Le Traquenard $^{5}(. .$.$) . Il a été poignardé pendant un sommeil réparateur après$ qu'il a profané le vagin de Sophie» (Bonono, 2007: 87). Si l'option pour la violence conduit Sophie en prison, force est de relever que son acte débouche sur sa relaxation à l'amiable. Craignant d'être dénoncé pour ses pratiques odieuses à travers le pays l'époux cède au chantage et vide son harem. Le texte des féministes apparaît finalement comme un plaidoyer pour une vision plus pacifique des rapports de sexe: le constructionnisme.

\subsection{Plaidoyer pour une vision constructionniste des rapports de sexe}

En affirmant qu'«on ne naît pas femme: on le devient. Aucun destin biologique, psychique, économique ne définit la figure que revêt au sein de la société la femelle humaine» (Beauvoir, 1949: 285), Beauvoir entendait mettre en avant l'idée du constructionnisme. Loin de se laisser abattre par une pratique qui l'avilit, la femme travaille au contraire dans le sens d'infléchir le sens de la courbe. Dans La Poupée Ashanti, Edna abandonne les bancs et son instituteur-violeur et se reconvertit dans les activités du marché auprès de sa grand-mère. Elle témoigne de ce que la

${ }^{5}$ C'est la narratrice qui souligne 
femme a droit d'aimer qui elle veut et quand elle le désire. C'est pourquoi, une fois majeure, Edna tombe amoureuse de Spio et soumet ce dernier à l'épreuve avant finalement d'en faire son âme sœur. De même, Fanny, dans Sous la cendre le feu, réalise sa naïveté et le poids de l'interdit. Son message est simple: la jeune fille réclame plus de regard face aux tabous sexuels qui l'exposent finalement au viol parental. II est indispensable pour sa saine croissance qu'elle reçoive une éducation sexuelle dépouillée de tout a priori, une éducation qui l'aide à prévenir toute catastrophe future. Mina, sa mère, se désole ainsi de ne l'avoir compris que trop tardivement: "dans nos mœurs, ce tabou qui frappe tout sujet relatif à l'intimité et à la sexualité est tel que les plus belles résolutions tombent quand il s'agit d'exprimer ces choses devant un enfant» (Mpoudi Ngolle, 1990: 194). Pourtant, l'aveu de Fanny aide sa mère Mina à récupérer un époux qui commençait à voguer sur le chemin de la perdition, à travers la pratique sectaire et l'usage de maléfices. Fleur brisée offre un socle au constructionnisme lorsque Francine parvient à tourner la page de son viol et se reconvertit en conseiller afin de soulager les femmes qui, comme elle, tombent sous le coup de la violence sexuelle.

Autant le dire, toutes les héroïnes des romans étudiés militent pour une transformation du regard condescendant qui pèse sur la femme car celle-ci n'est pas «l'inessentiel en face de l'essentiel (...) l'inessentiel qui ne retourne jamais à l'essentiel; comme l'autre absolu, sans réciprocité» (De Beauvoir, 1949: 52). Les constructionnistes sont des antinaturalistes parce qu'ils soutiennent que la culture précède l'essence ou la nature. Pour eux,les différences constatées entre hommes et femmes sont, pour une grande part, construites par un conditionnement social. A cet égard, «il n'existe pas d'essence féminine ou masculine mais unsexe biologique qui n'influe pas ou très peu sur la personnalité» (Nnomo, 2013: 23).

L'écrivain féministe contribue ainsi à la redéfinition du féminisme. II ne s'agit plus pour elle de valoriser les expériences et les idées des femmes aveuglément soumises, mais de soutenir le postulat neuf qui caractérise désormais la prestation en tant que féministes. Pour eux, «les hommes et les femmes devraient être égaux, socialement, économiquement et juridiquement» (Matlin, 2007: 12). Le féminisme change donc de combat ou, si l'on veut, renouvelle les termes de son combat en vue de devenir culturel. Les romanciers étudiés se positionnent comme des féministes culturels en ceci qu'ils militent pour une société «restructurée de façon à accentuer la coopération et non l'agression» (Matlin, 2007: 13). II s'agit in fine d'un renouvellement des rapports sociaux de sexe, en vue de la célébration de la femme qui «combattrait la dévalorisation des femmes, en reconnaissant la différence de genre et en réévaluant la féminité» (Fraser, 2005: 32).

\section{Conclusion}

Au total, le roman féministe se dévoile comme le roman de la militance par excellence, à plus d'un titre. II accompagne la femme violentée, la femme sexuellement agressée, à travers les arcanes et les 
strates successives de sa renaissance sociale, depuis son ostracisation par le mâle dominant, jusqu'à sa reprise en main et son repositionnement comme être de droits. Voilà pourquoi le romancier féministe ne prête pas le flanc à la riposte violente visant à contrecarrer l'action de l'homme phallocratique. II s'agit pour les romanciers de postuler un type nouveau de rapports entre les sexes, en générant une femme neuve et un homme neuf dans une cité tout aussi rénovée. Les rapports neufs dont il est question dessinent le tracé d'un nouveau monde d'équité au sein duquel la femme est respectée comme créature humaine, au même titre que l'homme, dans le cadre d'échanges intersubjectifs fructueux plus dignes. Autant le croire, l'agression sexuelle s'avère une des formes les plus horribles de violence parce qu'elle avilit la race humaine tout entière et jette l'opprobre sur l'homme-violeur. Elle concoure à la réification de la femme et hypothèque l'avènement d'un monde harmonieux où hommes et femmes cheminent ensemble vers une cité réconciliée avec elle-même. II apparaît à cet égard que la littérature se déploie véritablement comme «la somme des réponses possibles aux questions réelles que se posent un homme et, à travers lui, une époque, une civilisation et, à la limite, l'humanité» (Doubrovsky, 1966: 93).

\section{Références Bibliographiques}

AhANDA-Essomba, Honoré-Godefroy. Le Fruit défendu. Yaoundé: CLE, 1975.

BARBERIS, Pierre. "La Sociocritique». In Méthodes critiques pour l'analyse littéraire, edited by Daniel Bergez, Pierre Barbéris, Pierre-Marc de Biasi, Luc Fraisse, Marcelle Marini, Gisèle Valency. Paris: Nathan, 1990, pp. 151- 182.

BARTHES, Roland. Essais critiques. Paris: Seuil, 1964.

BEBEY, Francis. La Poupée Ashanti. Yaoundé: CLE, 1973.

BEYALA, Calixthe. Lettre d'une Africaine à ses sœurs occidentales. Paris: Spengler, 1995.

Bonono, Angéline Solange. Le Journal intime d'une épouse. Yaoundé: SOPECAM, 2007.

Bugul, Ken. Le Baobab fou. Paris: Présence Africaine, 2009.

CHesneau, Thierry. Basic Einstein. Paris: Mon Petit éditeur, 2010.

CheVRIER, Jacques. L'Arbre à palabres. Paris: Hatier, 1989.

CROS, Edmond. Sociocritique. Paris: L'Harmattan, 2003.

BEAUVoIR, Simone de. Le Deuxième sexe. Paris: Gallimard, 1949.

Doubrovsky, Serges. Pourquoi la nouvelle critique? Critique et objectivité. Paris: Mercure de France, 1966.

FORCOLIN, Francesca. «François Harvey, Alain Robbe-Grillet: le nouveau roman composite. Intergénéricité et intermédialité». InStudi Francesi, 167 (LVI | II)|2012, pp. 366-367.

FRASER, Nancy. «Multiculturalisme, anti-essentialisme et démocratie radicale: genèse de l'impasse actuelle de la théorie féministe». In

Cahiers du Genre. Paris: L'Harmattan, 2005, pp 31-45.

KRAFFT-EbING, Richard Von. Psychopathia sexualis. Bloat Books, 1999. 
KRAUSS, Cynthia. "AAvarice épistémique"» et économie de la croissance: le pas rien du constructionnisme social». In Le Corps, entre sexe et genre, edited by Hélène Rouch, Elsa Dorlin, Dominique Fougeyrollas Schwebel. Paris: L'Harmattan, 2005, pp. 39-42.

MATLIN, Margaret. Psychologie des femmes. Bruxelles: De Boeck, 2007.

MITTERAND, Henri. Le Discours du roman. Paris: PUF, 1980.

Mpoudi NGolLe, Evelyne. Sous la cendre le feu. Paris: L'Harmattan, 1990.

N'GUeSSAN KouAdIO, Germain. Féminisme: (en)jeux d'une théorie. Abidjan: Inidaf, 2017.

NGo IBOUM, Francine. Fleur brisée. Paris: L'Harmattan, 2011.

NNOMO, Marcelline. "Le genre: de la pensée de la différence à la postulation d'une catégorie d'analyse». In Heuristique, Vol. 1, $\mathrm{n}^{\circ} 2$, 2013, pp. 11-25.

ONOMO Abena, Sosthène. «EI reencuentro. El retorno de un exiliado de Juan Balboa Boneke. Un roman-témoignage de dénonciation». In Revue Syllabus, Vol. 1, $n^{\circ}$ 6. Yaoundé: PUY, 1998, pp. 81-98.

SADAMBA, Tcha Koura. Femme infidèle. Lomé-Dakar-Abidjan: NEA, 1988. 
Ambigua, Revista de Investigaciones sobre Género y Estudios

Culturales, n. ${ }^{\circ}$ 7, 2020, pp. 146-163. ISSN: 2386-8708 